\title{
Effects of User Reviews and Critic Rating on Online Healthcare Sales
}

\author{
Wenbo Han \\ Robert H. Smith School of Business, University of Maryland, College Park, USA \\ Email: weh15107@umd.edu
}

How to cite this paper: Han, W. B. (2020). Effects of User Reviews and Critic Rating on Online Healthcare Sales. American Journal of Industrial and Business Management, 10, 1902-1915.

https://doi.org/10.4236/ajibm.2020.1012119

Received: November 26, 2020

Accepted: December 28, 2020

Published: December 31, 2020

Copyright $\odot 2020$ by author(s) and Scientific Research Publishing Inc. This work is licensed under the Creative Commons Attribution International License (CC BY 4.0).

http://creativecommons.org/licenses/by/4.0/ Open Access

\begin{abstract}
The value of online healthcare has been well documented by academicians and practitioners. Nevertheless, prior literature has paid little attention to the impacts of user reviews and critic rating of doctors in patients' purchase decision. In this research, we empirically examine the effects of user reviews and critic rating of doctors on online healthcare sales with secondary data on 3283 doctors from Haodaifu.com in China. The results show that both user review valence and volume have positive effects on online healthcare sales. User review volume can amplify the positive effects of review valence on online healthcare sales, since it is an extrinsic, high-scope cue in patients' decision-making that can alter the diagnosticity of review valence. We further find that the critic rating of doctors can weaken the positive of user review valence and volume on online healthcare sales. We also build a utility model to extend and validate the above findings.
\end{abstract}

\section{Keywords}

Online Healthcare, User Reviews, Critic Rating of Doctors, Haodaifu

\section{Introduction}

Online healthcare websites (e.g., haodaifu, RateMDs etc.) have been gaining popularity among patients in the world, and have attracted considerable interest among researchers (Yang, Zhang, \& Lee, 2019; Zhang et al., 2017). These websites enable patients to undertake the preliminary online as well as regular health consultations. At healthcare websites, patients can search for appropriate medical doctors (both general practitioners and specialists), describe their symptoms to them, and upload the related medical test results. The selected doctor then assesses the patient's well-being and advises him/her on how to self-manage 
his/her medical afflictions.

Some research has offered evidence that online healthcare can help alleviate many of the critical challenges being faced by hospitals, e.g., limited capacity, long queues, and geographic inconvenience (Gummerus et al., 2004, Griffiths et al., 2006, Lu et al., 2011, Johnston et al., 2013). However, because online healthcare services are important to patients' health and are new to patients, the purchase rate of online healthcare services is very low, for example, in China, the purchase rate of online healthcare services is far less than the purchase rate of e-commerce (Yang, Wang, \& Mei, 2020).

Online healthcare services purchase involves a high level of uncertainty. Little information is available for patients to assess the service quality of the doctors, making it difficult to decide whether to make the purchase (Bonifield, Cole, \& Schultz, 2010; Kostyk, Niculescu, \& Leonhardt, 2017). During this stage, online reviews of prior users and critical rating of doctors become helpful information sources for patients to evaluate the healthcare service. When reviewing the healthcare service of a doctor, patients can access both the comments and numerical ratings of prior users, as well as critical rating of doctors. Online healthcare websites, such as Haodaifu.com ("Good Doctor Online" in Chinese), typically summarize the numerical ratings by presenting the average ratings (i.e., user review valence) and the number of reviews (i.e., user review volume) on the homepage of doctors. Very limited amount of empirical research has investigated the effects of user reviews and critical rating of doctors on online healthcare sales-an area that is critical for online healthcare providers to increase sales. For this purpose, we empirically investigate the effects of user reviews and critical rating of doctors on online healthcare sales in the context of Haodaifu.com, a leading online healthcare platform in China. Additionally, we also build a utility model to extend and validate the findings from the empirical study.

The remainder of this paper is structured as follows. In the next section, we develop the research hypotheses. Then, we follow the hypotheses with empirical analyses and findings. We also build a utility model to extend and validate the above findings. Finally, we conclude the paper with a discussion of the theoretical and managerial implications as well as limitations and suggestions for future research.

\section{Hypotheses Development}

To better understand the relationship among user reviews, critic rating of doctors and online healthcare sales, we develop a conceptual framework (see Figure 1). We propose that user review valence and volume have positive effects on online healthcare sales. Further, we predict that user review volume can amplify the positive effects of user review valence on online healthcare sales. However, critic rating of doctors can mitigate the positive effects of user review valence and volume on online healthcare sales. Below, we elaborate on each concept and present the hypotheses. 


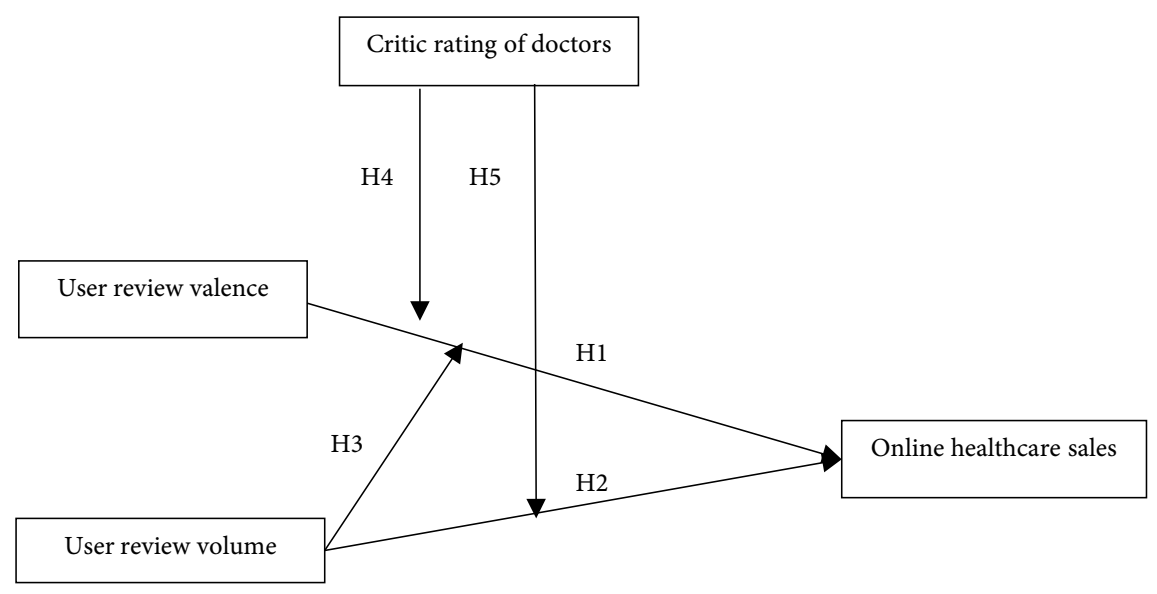

Figure 1. Overview of the conceptual framework.

In online situations, consumers do not know the true quality of products when making their purchase decisions. Similarly, in the context of online healthcare, it is more asymmetric between patients (consumers) and doctors (service providers). Therefore, patients rely on multiple service quality cues to make the judgement of a purchase (e.g., Rao \& Monroe, 1988). Prior research on marketing, related to cue diagnosticity framework, has classified these cues as being either intrinsic or extrinsic to the product (Miyazaki, Grewal, \& Goodstein, 2005). Intrinsic cues are related to physical attributes of a product (e.g. weight, material), while extrinsic cues represent the product but are not part of the product itself (e.g. price, brand). Online purchasing is a setting with high uncertainty. Patients cannot tell the service quality by intrinsic cues, which makes extrinsic cues, for example, user reviews, more important in the context of online healthcare community.

User reviews are on websites that prior buyers rate (usually with star ratings) and make comments on the basis of their personal usage experience and preferences (Appelt, 2010; Chen \& Xie, 2005; Moon, Bergey, \& Iacobucci, 2010). For instance, an online healthcare website-Haodaifu-presents ratings of prior user reviews and the comments on the healthcare service. In online healthcare setting, user reviews can serve as extrinsic cues of the healthcare service quality, and have significant impacts on patients' decision making and online healthcare sales (e.g. Godes \& Mayzlin, 2004; Srinivasan, Anderson, \& Ponnavolu, 2002). Two characteristics of user reviews have been explored in the extant research: user review valence, the preference carried in the review information (Duan, $\mathrm{Gu}$, \& Whinston, 2008), and user review volume, the number of reviews or ratings (Basuroy, Chatterjee, \& Ravid, 2003; Chevalier \& Mayzlin, 2006). In this section, we propose that these two characteristics of user reviews at healthcare websites can increase the sales of online healthcare.

\subsection{Effect of User Review Valence on Online Healthcare Sales}

Review valence indicates the favorability of the product (Moe \& Trusov, 2011). 
A majority of existing research has examined the impacts of review valence and a general consensus holds that positive review valence can enhance product sales, while negative valence decreases sales (Chevalier \& Mayzlin, 2006; Moon, Bergey, \& Iacobucci, 2010). As indicated by the attribution model of persuasion (Wood \& Eagly, 1981), user reviews message can help shape readers' expectations about the quality and performance of the product. Applying the above arguments in online healthcare context, when user review valence of a doctor is high, patients would form higher expectations and perceive the healthcare of the doctor to be more high-quality. Thus, the high expectations are more likely with high user review valence, which can increase the sales of online healthcare. We hypothesize the following:

H1: user review valence has a positive effect on online healthcare sales.

\subsection{Effect of User Review Volume on Online Healthcare Sales}

Review volume is the number of reviews that prior buyers posted on a product or service. High review volume can increase the exposure of a business or product offering. Much existing research has also examined the effectiveness of review volume in driving consumer purchase decisions and product sales (Kostyra, Reiner, Natter, \& Klapper, 2016; Zhu \& Zhang, 2010). Hasher \& Zacks (1984) demonstrate that information about numbers or counts are easy to process, since it is a primary way to store information. Moreover, an opinion expressed by more people indicates the popularity of the opinion and conveys the correctness (Weaver, Garcia, Schwarz, \& Miller, 2007; Baker \& Petty, 1994). In marketing research, Coulter \& Roggeveen (2012) has shown that the number of deals purchased does indeed influence consumer evaluation. Consequently, the higher user review volume is, the more positive evaluation of the doctor is. The higher positive evaluations can increase the expectations of patients on the healthcare compared to the same doctor having lower user review volume. As a result, review volume increases the likelihood of patients purchase. We thus posit:

H2: User review volume has a positive effect on online healthcare sales.

\subsection{Amplification Effects of User Review Volume}

The cue diagnosticity framework (Miyazaki, Grewal, \& Goodstein, 2005; Purohit \& Srivastava, 2001), suggests that, when confronted with multiple cues, consumers will use relatively more diagnostic cues to perceive the quality of the product belongs. Purohit \& Srivastava (2001) have extended this argument by proposing that the diagnosticity of a particular cue types depends on the valence of other cue types. Realistically, a particular cue may seem to be more diagnostic than another (Lynch, 2006; Milgrom \& Roberts, 1986). Similarly, in line with cue diagnosticity framework, a high-scope cue can alter the perceived diagnosticity of a low-scope cue. Khare, Labrecque, \& Asare (2011) highlight online review volume as an extrinsic, high-scope cue in consumer decision-making. Based on the literature above, we predict the interactive effects that user review volume can alter the diagnosticity of user review valence, due to its high-scope status. 
As we argued above, a positively valenced review increases patients preference for the review targeted healthcare service, and then lead to high level of expectations, which can increase the online healthcare purchase. Due to the greater diagnosticity of user review volume, the effect of user review valence on online healthcare sales should be greater when user review volume is higher. This is because the overall rating converges toward the true value as the volume of review increases (Ho-Dac et al., 2013; Zhu \& Zhang, 2010). The higher user review volume is, the more trustworthy review valence becomes. Therefore, high-volume review should strengthen the positive evaluation of the product and improve the level of expectations, which can increase healthcare sales. We hypothesize the following:

H3: User review volume positively moderates the effect of user review valence on online healthcare sales.

\subsection{Mitigation Effects of CRITIC Rating of Doctors}

Critic rating is provided by a third-party professional organization with professional knowledge besides the buyers and sellers (West \& Broniarczyk, 1998). When patients browse online healthcare websites, the critic rating of doctors is usually displayed on the homepage of doctors to help patients make purchase decisions. As online healthcare is a special service offering, which is important for patients' health, patients will be more cautious in the decision-making process of purchasing online healthcare. Therefore, when choosing online healthcare services, patients will pay more attention to the critic rating of doctors while browsing the prior user reviews. When the critic rating of doctors is higher, even though user review valence is negative or level of user review volume is low, patients still choose the doctor with high critic rating to avoid potential health risks. People generally believe that doctors with a high critic rating will provide more reliable health care service, thus, they still choose to buy the services from the doctors with high critic rating. In other words, the critic rating of doctors weakens the positive effect of user review valence and volume on patients' purchase of online healthcare services. Therefore, the following hypothesis is proposed:

H4: Critic rating of doctors negatively moderates the effect of user review valence on online healthcare sales.

H5: Critic rating of doctors negatively moderates the effect of user review volume on online healthcare sales.

On the basis of the above theoretical statements, the conceptual framework (Figure 1) is developed. In Study 1, we firstly examined the hypotheses, using secondary data from Haodaifu.com in China. In the second study, a utility model was designed to extend and validate the findings of Study 1.

\section{Study 1: Empirical Study}

In Study 1, we examine the effects of user review valence and review volume on 
online healthcare sales, as well as the moderation effects of user review volume and critic rating of doctors. To test the relationships, we use secondary data from Haodaifu.com in China.

\subsection{Data Collection and Sample}

The data for this research come from Haodaifu.com, the largest online healthcare community in China. Haodaifu was founded in 2006. After more than 10 years' development, it has developed into a comprehensive healthcare website, which integrates graphic and text consultation, telephone consultation, appointment and referral, remote outpatient service, post-diagnosis management, family doctor and other fields. Until December 2019, there are 610,000 doctors from 9917 hospitals across the country at Haodaifu. At Haodaifu, 225,000 doctors from the best hospitals in China (Baike, 2020), that can offer patients authoritative and reliable healthcare services. As a result, many patients in China gradually purchase online healthcare services from the doctors at Haodaifu.

After purchasing healthcare service at Haodaifu, patients can rate the doctor based on their experience. With Haodaifu, we have access to detailed information about doctors' sales, user reviews, and critic rating of doctors. Such access is consistent with our interest in studying the effects of user reviews and critic rating of doctors on online healthcare sales. We programmed a web crawler to search and collect data from Haodaifu, from June 20, 2018 to June 27, 2018. A total of 5125 doctors' sales data of online healthcare services were captured. We have deleted the record that the sales volume is null and zero. Due to most doctors at Haodaifu come from the best hospitals in China, thus, we eliminated the doctors data from other hospitals to rule out the effects of hospital level. The final sample is 3283 doctors, the data from which are used to test the effects of user reviews and critic rating of doctors on online healthcare sales.

\subsection{Measurement}

The definitions and operationalization associated with the measures are presented in Table 1. In regard to the operationalization of the variables, first, the dependent variable of interest is online healthcare sales, measured as the number of patients treated by a doctor within last two weeks. Second, Haodaifu makes publicly available the score of a doctor rated by patients who have purchased his services. We use the score measure user review valence. Third, Haodaifu provides details on total reviews. We measure user review volume as the number of reviews of a doctor. Fourth, Critic rating of doctors is operationalized as the doctor title. We divided doctor title into resident doctor, attending physician, deputy chief physician, and chief physician. Finally, this research includes hospital type and doctor activity as control variables to rule out potential explanations. At present, hospitals in China can be divided into two types: specialized hospitals and general hospitals. General hospitals are divided into more detailed 
departments, such as surgery orthopedics, chest, extracranial, urology, etc., while specialized hospitals usually focus only one disease. If the doctors are active on the website which can increase their popularity, and thus, the sales of the doctor will increase. To eliminate this effect, we also control the impact of doctor activity on the results of data analysis. Doctors at Haodaifu usually publish medical article, soul chicken soup or their own academic research on their homepage to help patients gain more disease-related knowledge, which also increases their visibility. We rule out this effect by including activity of doctors. In this research, we use the total number of articles posted on Haodaifu.com to measure the activity of doctors on healthcare websites. Table 2 provides summary statistics and correlation matrix for all variables. The average sale of online doctors is 64.50. The average of user review valence, user review valence, and critic rating of doctors are 4.14, 99.18, and 3.23, respectively. Among the correlation matrix, we can find that user review valence and user review valence volume have a large correlation. Not surprisingly, buyers are more likely to purchase a product/service when there are more positive reviews.

Table 1. Variable definitions and operationalization.

\begin{tabular}{lll}
\hline Variable & Operationalization & Note \\
\hline copy & More table copy & \\
Online healthcare sales & $\begin{array}{l}\text { Number of patients treated by } \\
\text { the doctor within last two weeks }\end{array}$ & \\
User review valence & $\begin{array}{l}\text { The score of a doctor rated by } \\
\text { patients who have purchased his services }\end{array}$ & \\
User review volume & The number of user reviews & Resident physician $=1$, \\
aritic rating of doctors & The title of doctor & $\begin{array}{l}\text { attending physician }=2, \\
\text { deputy chief physician }=3, \\
\text { chief physician }=4\end{array}$ \\
& & The total number of articles \\
Activity of doctors & posted on Haodaifu.com by doctors & \\
& &
\end{tabular}

Table 2. Descriptive statistics and correlation matrix.

\begin{tabular}{lcccccccc}
\hline & Mean & SD & 1 & 2 & 3 & 4 & 5 & 6 \\
\hline 1. Online healthcare sales & 64.50 & 122.82 & - & & & & & \\
2. User review valence & 4.14 & 0.29 & 0.46 & - & & & \\
3. User review volume & 99.18 & 157.55 & 0.58 & 0.63 & - & & & \\
4. Hospital dummy & 0.74 & 0.44 & -0.10 & -0.16 & -0.09 & - & & \\
5. Activity of doctors & 17.46 & 76.42 & 0.17 & 0.14 & 0.21 & -0.03 & - & \\
6. Critic rating of doctors & 3.23 & 0.80 & 0.03 & 0.18 & 0.18 & -0.01 & 0.03 & - \\
\hline
\end{tabular}




\subsection{Hypothesis Testing}

We used ordinary least squares (OLS) regression models to estimate online healthcare sales. We propose that user review valence positively affects online healthcare sales, which is supported $(\beta=0.151, p=0.000)$. In addition, we find that user review volume significantly and positively affects online healthcare sales $(\beta=0.479, p=0.000)$. The estimates for the regression path model appear in Table 3.

Table 4 shows the test result of $\mathrm{H} 3$ through $\mathrm{H} 5$. The interaction between user review volume and user review valence on online healthcare sales is positive and significant $(\beta=0.069, p<0.05)$, indicating that user review volume has a positive moderating effect on the relationship between user review valence and online healthcare sales, consistent with $\mathrm{H} 3$. In $\mathrm{H} 4$, we propose that critic rating of doctors negatively moderates the relationship between user review valence and online healthcare sales, which is also supported $(\beta=-0.035, p<0.05)$. The interaction between user review volume and critic rating of doctors has a negative effect on online healthcare sales $(\beta=-0.116, p=0.000)$, which supports H5. This result demonstrates that critic rating of doctors weakens the positive effects of user reviews on the sales of doctor online. When doctors at healthcare websites have high level of critic rating, the relationship between user review and online healthcare sales is weaker than when critic rating of doctors is low.

Table 3. Hypothesis testing.

\begin{tabular}{llll}
\hline Hypothesis & Parameter Estimate & $\mathrm{t}$ & $p$ \\
\hline User review valence $\rightarrow$ Online healthcare sales & $0.151^{\star * *}$ & 8.552 & 0.000 \\
User review volume $\rightarrow$ Online healthcare sales & $0.479^{\star * *}$ & 26.773 & 0.000 \\
Activity of doctors $\rightarrow$ Online healthcare sales & $0.054^{* * *}$ & 3.617 & 0.000 \\
Hospital dummy $\rightarrow$ Online healthcare sales & $-0.043^{* *}$ & -2.939 & 0.003 \\
\hline
\end{tabular}

a. ${ }^{* *} p<0.01,{ }^{* *} p<0.001$.

Table 4. Analysis of moderating effect.

\begin{tabular}{|c|c|c|c|}
\hline Hypothesis & Parameter Estimate & $\mathrm{t}$ & $p$ \\
\hline User review valence $\rightarrow$ Online healthcare sales & $0.123^{\star \star \star}$ & 6.577 & 0.000 \\
\hline User review volume $\rightarrow$ Online healthcare sales & $0.507^{\star \star \star}$ & 17.864 & 0.000 \\
\hline $\begin{array}{l}\text { User review valence }{ }^{*} \text { User review volume } \rightarrow \\
\text { Online healthcare sales }\end{array}$ & $0.069^{*}$ & 2.543 & 0.011 \\
\hline $\begin{array}{l}\text { User review valence }{ }^{\star} \text { Critic rating of doctors } \rightarrow \\
\text { Online healthcare sales }\end{array}$ & $-0.035^{\star}$ & -1.982 & 0.048 \\
\hline $\begin{array}{l}\text { User review volume }{ }^{*} \text { Critic rating of doctors } \rightarrow \\
\text { Online healthcare sales }\end{array}$ & $-0.116^{\star * *}$ & -5.613 & 0.000 \\
\hline Critic rating of doctors $\rightarrow$ Online healthcare sales & $-0.090^{\star * *}$ & -6.089 & 0.000 \\
\hline Activity of doctors $\rightarrow$ Online healthcare sales & $0.044^{\star *}$ & 3.070 & 0.002 \\
\hline
\end{tabular}

a. ${ }^{\star} p<0.05,{ }^{* *} p<0.01,{ }^{* *} p<0.001$. 


\section{Study 2: Utility Model}

In Study 1, we find that both user review valence and volume available at the time of purchase has a significant positive effect on the probability of healthcare purchase, while critic rating of doctors weakens the positive effects of user reviews on the sales of doctor online. In Study 2, a utility model was designed to extend and validate the findings of Study 1.

Suppose that the total volume of patients who suffer from single type of disease is 1 . There are three types of doctors' critic rating that can be chosen by the patients, high level doctor, median level doctor and low level doctor, which are represented by subscripts $h, m, l$, respectively. We assume that all patients value higher level doctor more than lower level doctor. Each patient has a valuation of $\delta \theta$, and 0 for the healthcare service provided by high level doctor, median level doctor and low level doctor, respectively, where $\delta>1, \theta \geq 0$. The reason we take the perceived value of low level doctor equals 0 is that these doctors can barely satisfy the basic treatment of patients.

Patients are heterogeneous in their severity of illness, i.e., if the patients are severely ill, they are more likely to obtain high valuation for the healthcare service of doctor, and if the patients are slightly ill, they value the service less. We character this feature by denoting $\theta$ as a uniform distributed parameter, where $\theta \sim U[0,1]$.

Each type of doctor charge a price of $P_{h}, P_{m}, P_{l}$, to the patient. We assume that $P_{l}$ patients can be fully covered by the medical insurance, i.e., $P_{l}=0$, and the price of high level doctor is higher than medium level doctor. The price of choosing each type of doctor is usually set by the government and hospital which cannot be changed frequently, thus, we set the price as an exogenously given parameter.

The user reviews of doctor are published on the website. Each comment of the specific type of doctor can add an additional net utility to the patients who choose them. We assume that this additional net utility is noted as $\gamma_{h} c_{h}$ and $\gamma_{m} c_{m}$. We do not involve low level doctors in the effect of user reviews, because these doctors are usually randomly allocated to the patients and are not permanent. The parameters $c_{h}$ and $c_{l}$ represents the volume of user reviews for high and low doctors. As patients are more trusted with the doctor who has higher reviews valence, $\gamma_{h}$ and $\gamma_{m}$ are the coefficients that represent the extent that a comment of each type of patient influence patients' additional obtained utility. We assume that, on the website, patients do not discriminate the comments for the doctors' type, i.e., each review provide equally value to the patients $\gamma_{h}=\gamma_{m}=\gamma$.

Given the assumptions above, the patients have three options, the net utility each patient can obtain during the process of treatment is given by

$$
\begin{gathered}
U_{h}=\delta \theta-P_{h}+\gamma c_{h}, \\
U_{m}=\theta-P_{m}+\gamma c_{m}, \\
U_{l}=0 .
\end{gathered}
$$


It is straight forward that, the patients who have higher $\theta$ will choose high level doctor, the patients who have medium $\theta$ will choose medium level doctor, the patients who have lower $\theta$ will choose low level doctor.

To calculate the volume of patients who choose each type of doctor, i.e., the demand of each type of doctor, we need to figure out the type of the patients $\theta_{1}$ who is indifferent about choosing high and medium level doctor, i.e., $U_{s}=U_{n}$, and the type of patients $\theta_{2}$ who is indifferent about choosing medium and low level doctor, i.e., $U_{n}=0$. Solving these equations, we can obtain $\theta_{1}=\frac{P_{n}-P_{s}+\gamma\left(c_{s}-c_{n}\right)}{1-\delta}, \theta_{2}=P_{n}-c_{n} \gamma$. We denote the portion of patients who choose high level of doctor as $\lambda_{h}$ and the portion of patients who choose low level of doctor as $\lambda_{m}$. Thus, we have

$$
\begin{aligned}
& \lambda_{h}=P\left[U_{h} \geq \max \left(U_{m}, U_{i}\right)\right]=P\left[\theta_{1} \leq \theta \leq 1\right], \\
& \lambda_{m}=P\left[U_{m} \geq \max \left(U_{h}, U_{l}\right)\right]=P\left[\theta_{2} \leq \theta \leq \theta_{1}\right] .
\end{aligned}
$$

Next we can obtain the volume of patients who choose each type of doctor $Q_{h}$ and $Q_{m}$

$$
\begin{aligned}
& Q_{h}=1 \cdot \lambda_{h}=1+\frac{P_{m}-P_{h}+\gamma\left(c_{h}-c_{m}\right)}{1-\delta}, \\
& Q_{m}=1 \cdot \lambda_{m}=\frac{P_{h}-\delta P_{m}+\gamma\left(\delta c_{m}-c_{h}\right)}{\delta-1} .
\end{aligned}
$$

To analyze the influence of user review volume to patients' demand of each type of doctor, we solve the first-order derivative of $Q_{h}$ and $Q_{m}$ in respect of $c_{h}$ and $c_{m}$ respectively, which gives

$$
\frac{\partial Q_{h}}{\partial c_{h}}=\frac{\lambda}{\delta-1}, \frac{\partial Q_{m}}{\partial c_{m}}=\frac{\delta \lambda}{\delta-1}
$$

As $\delta>1, \frac{\partial Q_{h}}{\partial c_{h}}<\frac{\partial Q_{m}}{\partial c_{m}}$ apparently holds. Moreover, as $\frac{\partial Q_{m}}{\partial c_{m}} / \frac{\partial Q_{m}}{\partial c_{m}}=\frac{1}{\delta}$, the effect of user reviews to the demand of high and low doctor differs more severely as $\delta$ grows. The reason may be that, although the same review volume improve the same amount of obtained utility of the patients, the improvement is more evident for medium level doctor than high level doctor, i.e., $\frac{\gamma C_{s}}{\delta \theta-P_{s}}>\frac{\gamma c_{n}}{\theta-P_{n}}$. Therefore, patients are less sensitive to user reviews when they chose high level doctor.

\section{Discussion}

Very limited prior research has explored the effects of user reviews and critic rating of doctors on online healthcare sales. In context of Haodaifu, we found evidence from 3283 doctors' sale data that user review valence and volume can increase online healthcare sales. We conceptualize and demonstrate that these positive effects can be weakened by critic rating of doctors. When doctors at 
healthcare websites have high level of critic rating, the relationship between user reviews and online healthcare sales is weaker than when critic rating of doctors is low. We also found that user review volume can increase the positive effect of user review valence on online healthcare sales. In Study 2, a utility model was designed to extend and validate the above findings. Below, we discuss the theoretical contributions and managerial implications of our findings.

\subsection{Theoretical Contributions}

This research contributes to the literature in two ways. First, we hypothesize and find support that two characteristics of user reviews have positive relationships with online healthcare sales. These findings add to research on online reviews (e.g., Appelt, 2010; Duan, Gu, \& Whinston, 2008; Ho-Dac, Carson, \& Moore, 2013) by extending to online healthcare research context. Other research has predicted that the effects of online reviews on consumer purchase decision (Godes \& Mayzlin, 2004; Srinivasan, Anderson, \& Ponnavolu, 2002), the importance of online healthcare (Gummerus et al., 2004, Griffiths et al., 2006, Lu et al., 2011; Johnston et al., 2013), but that they are not motivated to test the effects of user reviews and critic rating of doctors on online healthcare sales. We predict and find support that user reviews and critic rating of doctors are critical in patients' purchase decision at healthcare websites.

Second, the analysis confirms that user review volume can amplify the effects of user review valance on online healthcare sales. These results reinforce the findings of Khare, Labrecque, \& Asare (2011) that suggest that the number elements can serve as an important extrinsic cue in consumers' decision. But we go beyond this research by suggesting that number of user reviews not only can increase patients' purchase intention, but also can alter the diagnosticity of user review valance. Khare, Labrecque, \& Asare (2011) have observed that wordof-mouth (WOM) volume can be as a high-scope, decision-making cue upon which the influence of other WOM-relevant characteristics on a WOM message's persuability depends. We found the same impact trend within our data. We contribute to the research of Khare, Labrecque, \& Asare (2011) by establishing the connections among the user review volume, user review valence, and online healthcare sales. As an extrinsic, high-scope cue (Purohit \& Srivastava, 2001), high-volume user reviews are more diagnostic than is low-volume user reviews for patients' decision-making. These findings also highlight the importance of understanding the role of number elements in online review research.

\subsection{Managerial Implications}

This research provides managerial insights for online healthcare providers. One important managerial implication of our research is that user sellers should be aware of importance of user reviews. This suggestion is helpful for the online doctors that who want to leverage positive user reviews to enhance sales and profitability. For example, some doctors at Haodaifu reward the users to en- 
courage them to post positive comments on their services. We find user review volume can amplify the effects of user review valence on online healthcare sales. Thus, this suggestion can be more useful for the online healthcare providers that have a large number of user reviews. Additionally, we suggest that online healthcare providers with low critic rating should pay more attention to user reviews. We find critic rating of doctors can weaken the positive effects of user review valence and volume on online healthcare sales. Patients still purchase the healthcare services from the doctors with high level of critic rating, even there is lower user review volume and some negative user reviews.

\subsection{Limitations and Directions for Further Research}

This research provides novel insights into user reviews and online healthcare purchase, though it still has several limitations that may be addressed by future research. First, we used data collected from Haodaifu.com to test our hypotheses. We encourage future research to use self-report data or behavioural experiments to further explore related research questions. Second, we test our hypotheses in the context of Haodaifu.com in China. We encourage future research to generalize these findings to other healthcare websites. Finally, due to data limitation, we could not assess the role of the review comments, which we believe is a promising avenue for further research.

\section{Acknowledgements}

The author thanks the AJIBM editor and review team for their helpful comments and suggestions for improvement.

\section{Conflicts of Interest}

The author declares no conflicts of interest regarding the publication of this paper.

\section{References}

Appelt, L. (2010). Literature Review: Online Consumer Product Ratings \& Reviews. Interaction and Interface Design, 1-13.

Baike (2020). Haodaifu.

https://baike.baidu.com/item/\%E5\%A5\%BD\%E5\%A4\%A7\%E5\%A4\%AB\%E5\%9C\%A8 \%E7\%BA\%BF/10400497?fr=aladdin

Baker, S. M., \& Petty, R. E. (1994). Majority and Minority Influence: Source-Position Imbalance as a Determinant of Message Scrutiny. Journal of Personality and Social Psychology, 67, 5. https://doi.org/10.1037/0022-3514.67.1.5

Basuroy, S., Chatterjee, S., \& Ravid, S. A. (2003). How Critical Are Critical Reviews? The Box Office Effects of Film Critics, Star Power, and Budgets. Journal of Marketing, 67, 103-117. https://doi.org/10.1509/jmkg.67.4.103.18692

Bonifield, C., Cole, C., \& Schultz, R. L. (2010). Product Returns on the Internet: A Case of Mixed Signals? Journal of Business Research, 63, 1058-1065.

https://doi.org/10.1016/j.jbusres.2008.12.009 
Chen, Y., \& Xie, J. (2005). Third-Party Product Review and Firm Marketing Strategy. Marketing Science, 24, 218-240. https://doi.org/10.1287/mksc.1040.0089

Chevalier, J. A., \& Mayzlin, D. (2006). The Effect of Word of Mouth on Sales: Online Book Reviews. Journal of Marketing Research, 43, 345-354.

https://doi.org/10.1509/jmkr.43.3.345

Coulter, K. S., \& Roggeveen, A. (2012). Deal or No Deal? How Number of Buyers, Purchase Limit, and Time-to-Expiration Impact Purchase Decisions on Group Buying Websites. Journal of Research in Interactive Marketing, 6, 78-95. https://doi.org/10.1108/17505931211265408

Duan, W., Gu, B., \& Whinston, A. B. (2008). The Dynamics of Online Word-of-Mouth and Product Sales-An Empirical Investigation of the Movie Industry. Journal of Retailing, 84, 233-242. https://doi.org/10.1016/j.jretai.2008.04.005

Godes, D., \& Mayzlin, D. (2004). Using Online Conversations to Study Word-of-Mouth Communication. Marketing Science, 23, 545-560.

https://doi.org/10.1287/mksc.1040.0071

Griffiths, F., Lindenmeyer, A., Powell, J., Lowe, P., \& Thorogood, M. (2006). Why Are Health Care Interventions Delivered over the Internet? A Systematic Review of the Published Literature. Journal of Medical Internet Research, 8, 67-76. https://doi.org/10.2196/jmir.8.2.e10

Gummerus, J., Liljander, V., Pura, M., \& Van Riel, A. (2004). Customer Loyalty to Content-Based Web Sites: The Case of an Online Health-Care Service. Journal of Services Marketing, 18, 175-186. https://doi.org/10.1108/08876040410536486

Hasher, L., \& Zacks, R. T. (1984). Automatic Processing of Fundamental Information: The Case of Frequency of Occurrence. American Psychologist, 39, 1372-1387. https://doi.org/10.1037/0003-066X.39.12.1372

Ho-Dac, N. N., Carson, S. J., \& Moore, W. L. (2013). The Effects of Positive and Negative Online Customer Reviews: Do Brand Strength and Category Maturity Matter? Journal of Marketing, 77, 37-53. https://doi.org/10.1509/jm.11.0011

Johnston, A. C., Worrell, J. L., Di Gangi, P. M., \& Wasko, M. (2013). Online Health Communities: An Assessment of the Influence of Participation on Patient Empowerment Outcomes. Information Technology \& People, 26, 213-235.

https://doi.org/10.1108/ITP-02-2013-0040

Khare, A., Labrecque, L. I., \& Asare, A. K. (2011). The Assimilative and Contrastive Effects of Word-of-Mouth Volume: An Experimental Examination of Online Consumer Ratings. Journal of Retailing, 87, 111-126. https://doi.org/10.1016/j.jretai.2011.01.005

Kostyk, A., Niculescu, M., \& Leonhardt, J. M. (2017). Less Is More: Online Consumer Ratings' Format Affects Purchase Intentions and Processing. Journal of Consumer Behaviour, 16, 434-441. https://doi.org/10.1002/cb.1643

Kostyra, D. S., Reiner, J., Natter, M., \& Klapper, D. (2016). Decomposing the Effects of Online Customer Reviews on Brand, Price, and Product Attributes. International Journal of Research in Marketing, 33, 11-26. https://doi.org/10.1016/j.ijresmar.2014.12.004

Lu, H. Y., Shaw, B. R., \& Gustafson, D. H. (2011). Online Health Consultation: Examining Uses of an Interactive Cancer Communication Tool by Low-Income Women with Breast Cancer. International Journal of Medical Informatics, 80, 518-528. https://doi.org/10.1016/j.ijmedinf.2011.03.011

Lynch Jr, J. G. (2006). Accessibility-Diagnosticity and the Multiple Pathway Anchoring and Adjustment Model. Journal of Consumer Research, 33, 25-27.

https://doi.org/10.1086/504129 
Milgrom, P., \& Roberts, J. (1986). Price and Advertising Signals of Product Quality. Journal of Political Economy, 94, 796-821. https://doi.org/10.1086/261408

Miyazaki, A. D., Grewal, D., \& Goodstein, R. C. (2005). The Effect of Multiple Extrinsic Cues on Quality Perceptions: A Matter of Consistency. Journal of Consumer Research, 32, 146-153. https://doi.org/10.1086/429606

Moe, W. W., \& Trusov, M. (2011). The Value of Social Dynamics in Online Product Ratings Forums. Journal of Marketing Research, 48, 444-456. https://doi.org/10.1509/jmkr.48.3.444

Moon, S., Bergey, P. K., \& Iacobucci, D. (2010). Dynamic Effects among Movie Ratings, Movie Revenues, and Viewer Satisfaction. Journal of Marketing, 74, 108-121. https://doi.org/10.1509/jmkg.74.1.108

Purohit, D., \& Srivastava, J. (2001). Effect of Manufacturer Reputation, Retailer Reputation, and Product Warranty on Consumer Judgments of Product Quality: A Cue Diagnosticity Framework. Journal of Consumer Psychology, 10, 123-134. https://doi.org/10.1207/s15327663jcp1003 1

Rao, A. R., \& Monroe, K. B. (1988). The Moderating Effect of Prior Knowledge on Cue Utilization in Product Evaluations. Journal of Consumer Research, 15, 253-264. https://doi.org/10.1086/209162

Srinivasan, S. S., Anderson, R., \& Ponnavolu, K. (2002). Customer Loyalty in e-Commerce: An Exploration of Its Antecedents and Consequences. Journal of Retailing, 78, 41-50. https://doi.org/10.1016/S0022-4359(01)00065-3

Weaver, K., Garcia, S. M., Schwarz, N., \& Miller, D. T. (2007). Inferring the Popularity of an Opinion from Its Familiarity: A Repetitive Voice Can Sound like a Chorus. Journal of Personality and Social Psychology, 92, 821-832. https://doi.org/10.1037/0022-3514.92.5.821

West, P. M., \& Broniarczyk, S. M. (1998). Integrating Multiple Opinions: The Role of Aspiration Level on Consumer Response to Critic Consensus. Journal of Consumer Research, 25, 38-51. https://doi.org/10.1086/209525

Wood, W., \& Eagly, A. H. (1981). Stages in the Analysis of Persuasive Messages: The Role of Causal Attributions and Message Comprehension. Journal of Personality and Social Psychology, 40, 246-254. https://doi.org/10.1037/0022-3514.40.2.246

Yang, D., Wang, Y., \& Mei, S. (2020). How to Balance Online Healthcare Platforms and Offline Systems? A Supply Chain Management Perspective. Managerial and Decision Economics, 3, 345-366. https://doi.org/10.1002/mde.3250

Yang, Y., Zhang, X., \& Lee, P. K. (2019). Improving the Effectiveness of Online Healthcare Platforms: An Empirical Study with Multi-Period Patient-Doctor Consultation Data. International Journal of Production Economics, 207, 70-80.

https://doi.org/10.1016/j.ijpe.2018.11.009

Zhang, Y., Qiu, M., Tsai, C. W., Hassan, M. M., \& Alamri, A. (2017). Health-CPS: Healthcare Cyber-Physical System Assisted by Cloud and Big Data. IEEE Systems Journal, 11, 88-95. https://doi.org/10.1109/ISYST.2015.2460747

Zhu, F., \& Zhang, X. (2010). Impact of Online Consumer Reviews on Sales: The Moderating Role of Product and Consumer Characteristics. Journal of Marketing, 74, 133-148. https://doi.org/10.1509/jm.74.2.133 